\title{
Energy Detection for MIMO Decision Fusion in Underwater Sensor Network: Critical Review
}

\author{
Shweta \\ Department of Electronics \& \\ Communication Engineering \\ KIET Group of Institutions, \\ Ghaziabad, U.P, India
}

\author{
Vibhav Kumar Sachan \\ Department of Electronics \& \\ Communication Engineering \\ KIET Group of Institutions, \\ Ghaziabad, U.P, India
}

\author{
Syed Akhtar Imam \\ Department of Electronics \& \\ Communication Engineering \\ Jamia Millia Islamia, New \\ Delhi, India
}

\begin{abstract}
Underwater sensor network has different applications ranging from environmental monitoring, data collection to survey mission and coastal surveillance. In this paper several fundamental aspects of underwater acoustic communication are discussed in detail. Different architecture and channel model are also been discussed. This paper also covers the latest techniques which are used in order to increase the data rate in underwater acoustic communication. The performance of the energy detector which is considered for binary hypothesis decision fusion has been reviewed and analyzed on different parameters of the investigation. This paper is based on a MIMO model for underwater acoustic network using Neymen-Pearson/ Bayesian hypothesis testing. Previous investigation and the conclusion will be useful for possible future research direction.
\end{abstract}

\section{Keywords}

Decision Fusion, Energy detection, Multiple-input Multiple-output (MIMO), underwater sensor networks

\section{INTRODUCTION}

Underwater sensor nodes will discover application in oceanographic information accumulation, pollution checking, offshore investigation, disaster prevention and tactical surveillance application. A few principle key parts of underwater acoustic communication system are researched [1]. As electromagnetic waves don't proliferate well underwater, acoustics assumes a key part in underwater communication. This can be classified as:

- Acoustic waves: This is the most flexible and broadly utilized technique as a part of underwater network because of the low attenuation (signal reduction) of sound in water. This is particularly valid in thermally steady profound water setting. On the other hand, the utilization of acoustic waves in shallow water can be unfavorably affected by temperature gradient, surface ambient noise, and multipath propagation because of reflection and refraction. The speed of acoustic wave in water is around $1500 \mathrm{~m} / \mathrm{s}$.

- Electromagnetic waves: On the front of utilizing EM waves as a part of radio frequencies, conventional radio does not function properly in underwater communication because of the conducting way of the medium, particularly on account of seawater..

- Free-space Optical (FSO) waves: These are used as wireless communication carriers and generally limited to very short separation due to the fact that serious waves absorption at the optical frequency band and solid backscatter from suspending particles. Even the clearest water has 1000 times the attenuation of pleasant air and turbid water has more than 100 times the attenuation of the thick mist [2]. The comparison between different underwater transmission media given in table 1 .

But the main challenge of acoustic communication is limited bandwidth, which is both frequency and range dependent, inter-symbol interference (ISI) occurs in underwater communication system [3].

Table 1: Comparison between different underwater transmission media

\begin{tabular}{|l|l|l|l|}
\hline & $\begin{array}{l}\text { EM } \\
\text { WAVE }\end{array}$ & $\begin{array}{l}\text { ACOUSTIC } \\
\text { WAVE }\end{array}$ & $\begin{array}{l}\text { OPTICAL } \\
\text { WAVE }\end{array}$ \\
\hline $\begin{array}{l}\text { Propagation } \\
\text { Speed }\end{array}$ & High & Very Slow & Very High \\
\hline Line of Sight & $\begin{array}{l}\text { Not } \\
\text { Required }\end{array}$ & $\begin{array}{l}\text { Not } \\
\text { Required }\end{array}$ & $\begin{array}{l}\text { Required } \\
\text { According } \\
\text { to Nodes }\end{array}$ \\
\hline $\begin{array}{l}\text { Impact on } \\
\text { Environment }\end{array}$ & Minimal & High & High \\
\hline $\begin{array}{l}\text { Achievable } \\
\text { Data Rate }\end{array}$ & High & Very Low & Very high \\
\hline $\begin{array}{l}\text { Network } \\
\text { Coverage }\end{array}$ & $\begin{array}{l}\text { Short } \\
\text { Range }\end{array}$ & $\begin{array}{l}\text { Very long } \\
\text { range }\end{array}$ & $\begin{array}{l}\text { Very Short } \\
\text { Range }\end{array}$ \\
\hline $\begin{array}{l}\text { Impact on } \\
\text { Marine Life }\end{array}$ & $\begin{array}{l}\text { Not } \\
\text { Known }\end{array}$ & Negative & Not Known \\
\hline
\end{tabular}

\subsection{Underwater Acoustic Sensors Networks Communication} Architecture and Channel Models Diverse models for two-dimensional and threedimensional underwater sensor network are itemized in this segment. Specifically, the present reference designs are two-dimensional, three-dimensional and a few types of autonomous underwater vehicles (AUVs) networks are used to upgrades the capacities of underwater sensor network. The accompanying structures are given as:

- Static two-dimensional UW-ASNs for sea base observing: These are constituted by sensor nodes that are anchored to the base of the sea. The application might be ecological observing or observing of underwater plates tectonics.

- $\quad$ Static three-dimensional UW-ASNs for ocean segment checking: In three dimensional underwater networks, sensors nodes float at various depths in order to 
monitor a given phenomena. One conceivable arrangement would be to attach each UW-sensor node to a surface float, by method for wires whose length can be managed in order to adjust the depth of every sensor node.

- $\quad$ Three-dimensional network of autonomous underwater vehicles (AUVs): These networks incorporate fixed portions composed of anchored sensors and mobile segment constitute via autonomous vehicles as detailed in [4].

Now, the term channel model might signify a physical propagation model, but on the other hand, it is utilized for scientific formulation of the channel impulse response. Channel models are imperative in different branches of communication research, including channel simulation, modulation and network scheme. The various channel models used to propagate acoustic wave in underwater communication system is explained in [5]. This may surmised in a table 5. However, modeling of underwater acoustic channel is still an area of research. Statistical model, include small-scale impacts scattering motioninduced Doppler shifting and in charge of quick varieties of the instantaneous channel reaction, while large-scale impact explain the area instability, changing ecological condition and influence the locally averaged received power [6].

\section{UNDERWATER ACOUCTIC COMMUNICATION AND NETWORKING}

Presently the high data rate in underwater communication has successfully brought about to design and analysis by using advanced techniques. In 2008 it was suggested that the channel having common Doppler scaling

Table 2: Comparison of different channel models with propagation effect

\begin{tabular}{|l|l|l|l|l|l|}
\hline & SNR & TINB & TVNB & MSML & UWB \\
\hline $\begin{array}{l}\text { Delay } \\
\text { Spread }\end{array}$ & NO & YES & YES & YES & YES \\
\hline $\begin{array}{l}\text { Doppler } \\
\text { Spread }\end{array}$ & NO & NO & YES & YES & YES \\
\hline $\begin{array}{l}\text { Freq. } \\
\text { Selective } \\
\text { Fading }\end{array}$ & NO & YES & YES & YES & YES \\
\hline $\begin{array}{l}\text { Freq. } \\
\text { Dependent } \\
\text { Path Loss }\end{array}$ & NO & NO & NO & NO & YES \\
\hline $\begin{array}{l}\text { Freq. } \\
\text { Dependent } \\
\text { fluct. Rate }\end{array}$ & NO & NO & NO & YES & YES \\
\hline Dispersion & NO & NO & NO & NO & YES \\
\hline
\end{tabular}

Element on all propagation ways, and propose a two stage way to deal with alleviating Doppler Effect:

1) Non-uniform Doppler compensation by means of re-sampling that changes over a "wideband" issue into a "narrowband" issue.

2) High-resolution uniform compensation of the remaining Doppler. The zero-padding orthogonal frequency division multiplexing (OFDM) is used to minimize the transmission power. Null subcarriers are utilized to encourage Doppler compensation and pilot subcarriers are utilized for channel estimation [7].

Later in 2009 a strategies was explained in which a multicarrier modulation scheme is introduced for acoustic communication at low signal-to-noise proportion (SNR). User bits are put through a rate $1 / 3$ turbo encoder and interleaved with periodic preparing bits. A maximal-length order is prefixed for signal detection and equalizer merging, and the subsequent bit stream is all the while modulated onto multiple phase-shift keyed carriers the same symbol arrangement, the baseband ensemble is amenable to multichannel equalization detailed in [8]. MIMO system in underwater communication is considered in [9], where spatial multiplexing is applied with orthogonal frequency division multiplexing (OFDM) signals.

Further in 2010 it was investigate that frequency domain oversampling to enhance the system execution of zeropadded OFDM transmissions over underwater acoustic channel with extensive Doppler spread is explained in [10]. Further, multicarrier communication system which transmits data rate is suggested in [11]. For this system, a passive-phase conjugation (PPC) based receiver structure is surveyed by preparing information gathered in ocean trials. On the basis on temporal diversity (pulse compression) misused by PPC preparing, an adaptive multichannel decision feedback equalizer is utilized to remove inter-symbol interference. The spatial diversity is abused by adaptive multichannel combination and on the basis of temporal diversity obtained by pulse compression. This proposed structure enhances the execution of the time reversal communication by adaptive diversity combining detailed in [12]. The capacity improvement is conceivable with the utilization of multiple-input multiple-output (MIMO) systems, MIMO communication is outline in shallow-water [13] by utilizing space-time coding at the transmitter, and MIMO decision feedback equalization at the receiver. Moreover, a soft input/soft output linear equalizer for Alamouti encoded MIMO signals are utilized to exploit the unpredictable and rich characteristics of the acoustic underwater channel in [14]. Now in [15], spatial modulation technique is utilized to increase the reliable data rate and received power in an underwater acoustic communication system.

Networking technologies and their designing principles apply in underwater acoustic networks have been considered in [16]. Energy-efficient multiple-access protocols suited for circumstances with long and obscure propagation delays were proposed and analyzed in [17] [19].The distinction in energy utilization between submerged acoustic and terrestrial radio device are investigated in [20]. Frequency and power allocation for underwater sensor network were designed in [21].

\section{DECISION FUSION}

In sensor network with countless sensors, a decision fusion rule utilizing the aggregate number of detection reported by local sensors for a final evaluation on a binary hypothesis testing. Based on signal attenuation model where the received signal power decays as the separation from the object expand, the system level identification acquired as probabilities of detection and false alarm. [22]. 


\section{State of phenomenon}

Source of uncertanity

Sensor network

Channel uncertanit

Fusion centre

Fig. 1: An illustration of sensor network with two layers of uncertainty

Distributed detection in wireless sensor network is still a dynamic zone for research. In underwater wireless sensor network, the design of distributed detection faces many challenges. From a system point of view, decision making in inference centric underwater sensor network is influenced by two level of uncertainty as show in fig 1 . The first level records for the disturbance and noise in the sensor observation. The second level of uncertainty is because of the transmission channel between the sensors and the fusion centre that are ordinarily influenced by receiver noise channel fading and interference. Several works are discussed in [23].

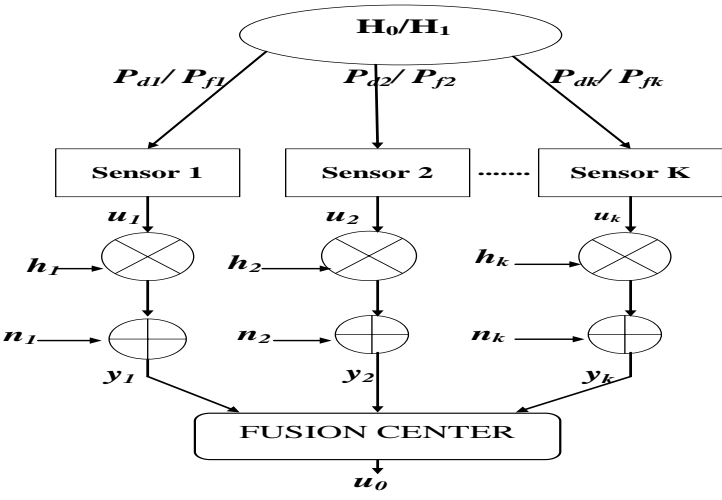

Fig. 2: Parallel Fusion Model

Information fusion utilized by multiple distributed sensors can be achieved using parallel access channel. Typical parallel fusion structure has various number of sensor observe information produced as $\mathrm{H}_{0}$ (target missing) or $\mathrm{H}_{1}$ (target present), which are the two hypotheses under test. Each sensor processes its observation and make on a preparatory decision about the hypothesis before sending it to decision fusion. Now, suppose that the $k t h$ local sensor accomplish a binary decision $u_{k} \in\{1,-1\}$, with the false alarm and detection probabilities $\mathrm{P}_{f k}$ and $\mathrm{P}_{d f}$, individually. That may be given as: $\mathrm{P}\left[u k=1 \mid H_{0}\right]=\mathrm{p}_{f k}$ and $\mathrm{P}\left[u k=1 \mid H_{1}\right]=\mathrm{p}_{d k}$. The figure 2 show, each local decision $u_{k}$ is forward through a fading channel and the outcome of the channel (input to the fusion centre) for the $k t h$ sensor is given as:

$y_{k}=h_{k} u_{k}+n_{k}$,
Where $h_{k}$ is real esteemed value of fading envelope with $h_{k}>0$, and $n_{k}$ is zero mean Gaussian noise with variance. This situation may consider numerous sensors that transmit their local decision to a fusion centre. The combination of the received information at the fusion centre provides global decision [24]-[25]. Differently, Near-optimal fusion rules with full channel state information (CSI) available at the receiver have been discussed in [26].

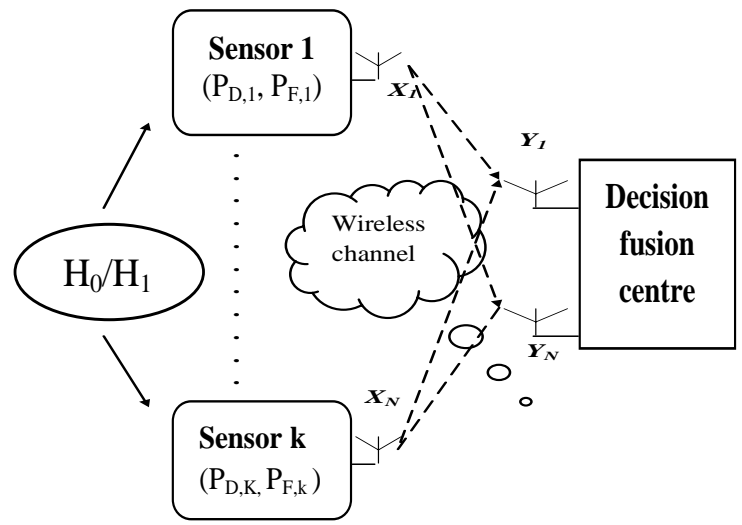

Fig. 3: The decision fusion model in presence of a (virtual) MIMO channel

The presence of multiple transmitters and receivers for distributed-detection causes interfering nature of wireless media. This can be resolve when the outcome is a communication over a "virtual" Multiple-input multipleoutput (MIMO) channel between the sensors and the decision fusion centre, as shown in figure 3.The impact of multiple antennas at the decision fusion centre has been researched by utilizing MIMO techniques and compared in terms of performance and complexity of system has been discussed [27]. Channel quality is measured through SNR present in [28]. Finally, a very simple receiver based on energy detection has been proven to be optimal (under Bayesian/ Neyman-Pearson system) in Rayleigh fading channels [29].

\section{A BRIEF SURVEY OF SYSTEM MODEL}

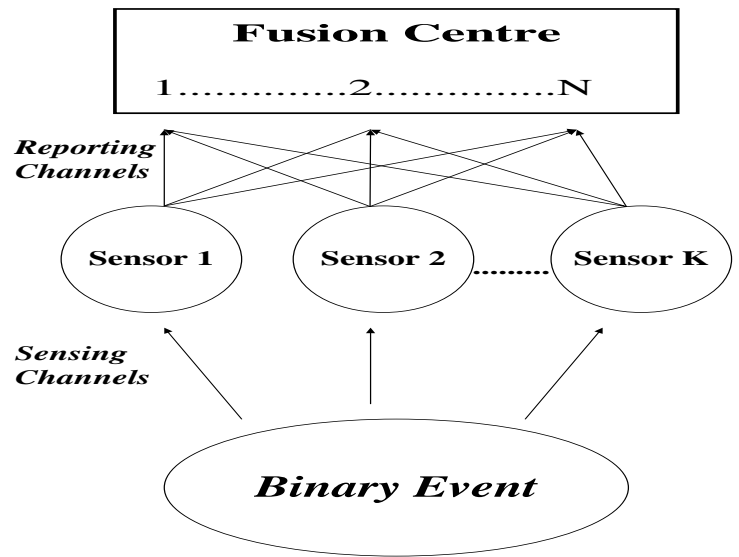

Fig. 4: Scenario for binary decision with $\mathrm{K}$ sensors and one fusion centre equipped with $\mathbf{N}$ hydrophones 
From the above give fig. 4, let considered the scenario in which $\mathrm{K}$ sensors sense independently the surroundings, each taking a local decision concerning a binary hypothesis test. The two hypotheses are denoted as $\mathrm{H}_{0}$ and $\mathrm{H}_{1}$ and the comparing from the a-priori probabilities $\pi_{0}$ and $\pi_{1}$, individually. It is consider that the local sensing and decision process by the $k t h$ sensor is completely depicted by the local probability of false alarm $p_{f}(k)$ and the local probability of missed detection $p_{m}(k)$ both thought to be stationary and conditionally autonomous provide the particular hypothesis. Sensors, each with one single acoustic transducer (projector), convey their decision to a fusion center, further with $\mathrm{N}$ hydrophones, whose point to give a robust decision on the premise of the multiple receive data. Every sensor utilizes the same binary modulation for energy saving purpose. Here, OOK modulation is considered with identical indistinguishable specification [30].

\subsection{Discrete-Time Signal Model}

Now, the signal transmitted by the $k$ th sensor, received at the fusion centre where the $m t h$ sample of received signal after sampling with frequency $f_{s}$ is given as:

$y_{n}[m]=\sum_{k=1}^{k} \mathrm{H}_{n, k}[m] x_{k}+w_{n}[m]$,

Where: $y_{n}[m]=y_{n}\left(m \mid f_{s}\right), w_{n}[m]=w_{n}\left(m \mid f_{s}\right)$ and

$$
\begin{aligned}
& H_{n . k}[m]=\sum_{l=1}^{L} \alpha_{l^{(n, k)}} e^{-j 2 \pi f_{c \tau} l_{l, k)}} e^{j 2 \pi \phi_{l^{(n, k) m}} \frac{f_{c}}{f_{s}}} \\
& \times g\left(\left(1+\phi_{l^{(n, k)}}\right) \frac{m}{f_{s}}-\tau_{l^{(n, k)}}\right)
\end{aligned}
$$

the above equation shows the received signal, the noise and the channel coefficient, individually. Define $y[\mathrm{~m}]=$ $\left(y_{1}[m], \ldots \ldots, y_{N}[m]\right)^{t}$ the vector gathering the signal at the $m$ th sampling time over all $\mathrm{N}$ hydrophones, $w[m]=$ $\left(w_{1}[m], \ldots, w_{N}[m]\right)^{t} \sim N_{c}\left(0_{N}, \sigma^{2} w \boldsymbol{I}_{N}\right)$ the

corresponding noise contribution, $x=\left(x_{1, \ldots . .,} x_{k}\right)^{t}$ represent the local decision from all the $\mathrm{k}$ sensors and

$$
\mathrm{H}[m]=\left(\begin{array}{ccc}
\mathrm{H}_{1.1}[m] & \cdots & \mathrm{H}_{1, \mathrm{k}}[m] \\
\vdots & \ddots & \vdots \\
\mathrm{H}_{\mathrm{N}, 1}[m] & \cdots & \mathrm{H}_{\mathrm{N}, \mathrm{K}}[m]
\end{array}\right)
$$

The above matrix detailed the channel coefficient at the $m t h$ sampling time. At that point the discrete-time model for the received signal at the $m t h$ sampling time is given as

$$
y[m]=\mathrm{H}[m] x+w[m]
$$

An integration time $T_{0}$ is defined as the collecting signals from $M=\left[f_{s} T_{0}\right]$ successive sampling times at define as: $y=\left(y[1]^{t}, \ldots \ldots, y[M]^{t}\right)^{t}, w=\left(w[1]^{t}, \ldots, w[M]^{t}\right]^{t}$, $H=\left(H[1]^{t}, \ldots, H[M]^{t}\right)^{t}$, provide the following discretetime model.

$$
y=H x+w
$$

Channel condition is measured through the proportion between the unitary energy of the active symbol and the noise variance, i.e. it is characterize the link SNR as follows

$$
\operatorname{SNR}=\frac{1}{\sigma_{\mathrm{w}}^{2}},
$$

\subsection{Decision Fusion}

The decision is normally executed as a test comparing a signal-dependent statistic $(\lambda(y))$ and a fixed threshold $(\gamma)$

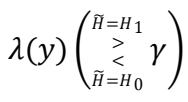

Where $\widetilde{H}$ signifies the estimated hypothesis. Execution is calculated in phrase of global probability of false alarm $\left(q_{f}\right)$ and global probability of missed detection $\left(q_{m}\right)$ is defined as follows

$$
\begin{aligned}
& q_{f}=p_{r}\left(\lambda(y)>\gamma \mid H_{0}\right), \\
& q_{m}=p_{r}\left(\lambda(y)>\gamma \mid H_{1}\right),
\end{aligned}
$$

Additional matter of interest is the global probability of $\operatorname{error}\left(q_{e}\right)$ is discussed as follows

$$
q_{e}=\pi_{0} q_{f}+\pi_{1} q_{m}
$$

The threshold in Eq. (8) is generally chosen in order to minimize the error probability (according to Bayes criterion [31]) or to provide a target probability of false alarm (according to the Neyman -person criterion [31]). For system execution assessment, in this paper it is examine the behavior of the global probability of missed detection $\left(q_{m}\right)$ versus the global probability of false alarm $\left(q_{f}\right)$, regularly meant complementary receiver operating characteristic (CROC). The log-likelihood ratio (LLR) of the obtained signal under the two hypotheses gives the ideal test (under both Bayesian/Neyman-Pearson [31])

$$
\begin{aligned}
& \lambda(y)=\log \left(\frac{p(y \mid \mathrm{H} 1)}{p(y \mid H 0)}\right) \\
= & \log \left(\frac{E_{H\left\{\sum x \varepsilon X^{k} p(y \mid \mathrm{H}, x) \Pi_{k=1}^{k} p r\left(x_{k} \mid \mathrm{H} 1\right)\right\}}}{\left.E_{H\left\{\sum x \varepsilon X^{k} p(y \mid \mathrm{H}, x) \Pi_{k=1}^{k} p r\left(x_{k} \mid H o\right)\right\}}\right),}\right. \\
= & \log \left(\frac{\sum x \in X^{k} E_{H}\left\{e^{-\frac{\|y-H x\|^{2}}{\sigma^{2} w}}\right\} \prod_{k=1}^{k} p r\left(x_{k} \mid \mathrm{H} 1\right)}{\sum x \in X^{k} E_{H}\left\{e^{-\frac{\|y-H x\|^{2}}{\sigma^{2} w}}\right\} \Pi_{k=1}^{k} \operatorname{pr}\left(x_{k} \mid \mathrm{H} 0\right)}\right),
\end{aligned}
$$

Now, from above equation several difficulties in optimal test has been discussed in [27]:

1) Computationally costly (complexity is exponential with $\mathrm{k}$ ).

2) High knowledge requirement of $\mathrm{H}, \mathrm{P}\left(x / H_{\mathrm{i}}\right)$ and $\sigma_{w}^{2}$

3) Numerically instability of the function, due to presence of exponential function.

On account on OOK, a typical less complex option is acquired supplanting the LLR with the energy of the received signal, i.e.

$\lambda(y)=\|y\|^{2}$,

Which obviously requires minimal computational complexity, furthermore has the point of preference that neither CSI nor SNR nor local sensor execution required. 
Such an analysis has been turned to be optimal in Rayleigh fading scenarios [32], [33]. In any case, in the accompanying section it is demonstrate how it can be an interesting test additionally in underwater acoustic channels. The observation bound is computed as follow in [27]:

$q_{f}=\sum_{l=c}^{k}\left(\begin{array}{l}k \\ l\end{array}\right) p_{f}^{l}\left(1-p_{f}\right)^{k-l}$,

$q_{m}=\sum_{l=0}^{c-1}\left(\begin{array}{l}k \\ l\end{array}\right)\left(1-p_{m}\right)^{l} p_{m}^{k-l}$,

Where is defined as $c \in\{0, \ldots, k\}$ is a discrete threshold. Now, this above equation can be analyzed the graph between optimal detector and energy detector. The following parameter is considered in [30] for the experiment in North Atlantic Ocean at water depth of $15 \mathrm{~m}$ and distance from transmitter and receiver elements between $60 \mathrm{~m}$ and $1000 \mathrm{~m}$. Parameters are assumed as follow: let considered a binary event with a-priori probability $\pi_{0}=0.7$ and $\pi_{1}=0.3$. Now, for $K=15$ transmitting sensor whose local sensing performance has been chosen as $P_{f}=0.04$ and $P_{m}=0.5$. Sensor transmit at carrier frequency $f_{c}=10 \mathrm{kHz}$ with $T_{p}=1 \mathrm{~ms}$. Up to $\mathrm{N}=4$ hydrophone at fusion centre, operating at sampling frequency $f_{s}=1 \mathrm{kHz}$ and integration time $T_{0}=10 \mathrm{~ms}$.

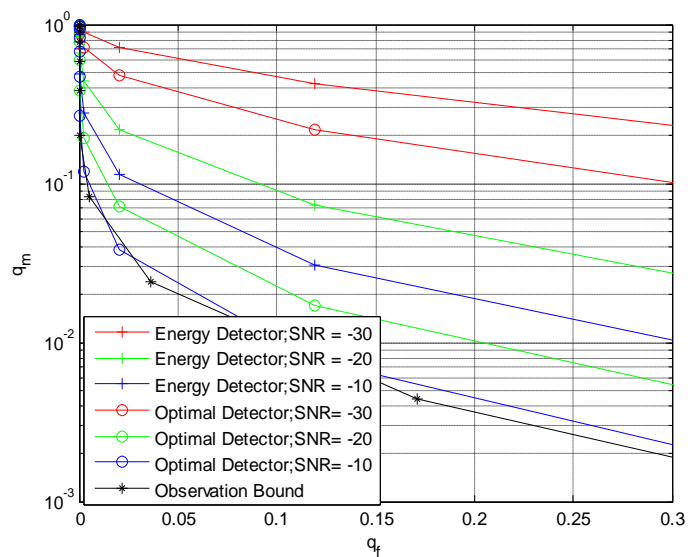

Fig.4: performance loss of energy detector with respect to optimal detector.

For different SNR the performance loss of energy detector regarding optimal detector is given in above figure 4 . The (ideal) observation bound is shown complete comparison. In order to assess the gap, It is shown that energy detector and optimal detector at global probability of false alarm $q_{f}=0.06$ with $\mathrm{SNR}=-20 \mathrm{~dB}$ gives global probability of missed detection of $q_{m}=0.15$ and $q_{m}=$ 0.049 respectively.

Now we can conclude that MIMO decision fusion based on energy detection is an engaging technique in underwater acoustic wireless sensor network since it is suboptimal. This can accomplish extremely good performance at low computational complexity and limited system knowledge.
Summary Table: 3

\begin{tabular}{|c|c|c|}
\hline TECHNIQUE & METHOD & RESULT \\
\hline $\begin{array}{l}\text { Multicarrier } \\
\text { modulation [6] }\end{array}$ & $\begin{array}{l}\text { Zero-padded } \\
\text { OFDM for } \\
\text { UWA channel }\end{array}$ & $\begin{array}{l}\text { Data rate for } \\
\text { QPSK= } \\
0.7,0.8,0.9 \mathrm{~kb} / \mathrm{s}\end{array}$ \\
\hline MIMO-OFDM [8] & $\begin{array}{l}\text { Zero-padded } \\
\text { OFDM }\end{array}$ & $\begin{array}{l}\text { Data rate for } \\
\text { 1)QPSK=31.4 } \\
\mathrm{kb} / \mathrm{s} \\
\text { 2) } 8 \mathrm{Q} A M=47.1 \\
\mathrm{~kb} / \mathrm{s} \\
\text { 3) } 16 \mathrm{QAM}=62.8 \\
\mathrm{~kb} / \mathrm{s}\end{array}$ \\
\hline $\begin{array}{l}\text { Adaptive turbo } \\
\text { multiband } \\
\text { equalization [7] }\end{array}$ & $\begin{array}{l}\text { Passive phase } \\
\text { conjugation } \\
\text { (PPC) }\end{array}$ & $\begin{array}{l}\mathrm{SNR}=-12 \mathrm{db} \\
\text { Data rate } \mathrm{R}=75 \\
\mathrm{~b} / \mathrm{s}\end{array}$ \\
\hline $\begin{array}{l}\text { Multichannel } \\
\text { communication [10] }\end{array}$ & $\begin{array}{lr}\text { Digital } & \text { phase- } \\
\text { locked } & \text { loop } \\
\text { (DPLL) } & \end{array}$ & $\begin{array}{l}\mathrm{SNR}=4.6 \mathrm{db} \\
\mathrm{BER}=0.024\end{array}$ \\
\hline $\begin{array}{l}\text { Spatial diversity in } \\
\text { multichannel [11] }\end{array}$ & $\begin{array}{l}\text { Passive Phase } \\
\text { conjugation } \\
\text { (PPC) }\end{array}$ & $\begin{array}{l}\mathrm{SNR}=2.2 \mathrm{db} \\
\mathrm{BER}=0.035 \\
0.01\end{array}$ \\
\hline
\end{tabular}

\section{CONCLUSION}

From last couple years developing in underwater acoustic communication become more and more interesting due to its application in marine research, oceanography, offshore oil industry, defense etc. This paper has reviewed the different technology of underwater acoustic network. Further, this paper concentrates to revise the energy detector for decision fusion in underwater acoustic wireless sensor networks over multiple access channels. More specifically, analytical performance in terms of global probability of false alarm and detection has been derived. Although not being optimal, the energy detector was selected for its low computational complexity and limited requirement on system knowledge. For future point of view of underwater sensor network, this might be use to setup a practical system that approach optimal performance in realistic scenario.

\section{REFERENCES}

[1] Ms. Akanksha V. Patil, Mrs. Sapana G. Buwa, Mr. Vijay U. Patil, "Underwater sensor network" IJSER.org, 2012.

[2] G. Hattab, M. El-Tarhuni, M. Al-Ali, T. Joudeh, N Qaddoumi, "An underwater wireless sensor network with realistic radio frequency path loss model", International Journal of Distributed Sensor Networks, vol 9, 2013.

[3] L. Lanbo, Z. Shengli, and C. Jun-Hong, "Prospects and problems of wireless communication for underwater sensor networks", Wireless Communication and Mobile Computing., vol. 8, no. 8, pp. 977-994, Oct. 2008.

[4] F. Akyildiz, D. Pompili, and T. Melodia, "Underwater acoustic sensor networks: Research challenges," Ad Hoc Network., vol. 3, no. 3, pp. 257-279, Mar. 2005.

[5] P. A. van Walree and R. Otnes, "Ultrawideband underwater acoustic communication channels," IEEE J. Ocean. Eng., vol. 38, no. 4, pp. 678-688, Oct. 2013. 
[6] P. Qarabaqi and M. Stojanovic, "Statistical characterization and computationally efficient modeling of a class of underwater acoustic communication channels," IEEE J. Ocean. Eng., vol. 38, no. 4, pp. 701-717, Oct. 2013.

[7] B. Li, S. Zhou, M. Stojanovic, L. Freitag, and P. Willett, "Multicarrier communication over underwater acoustic channels with non-uniform Doppler shifts," IEEE J. Ocean. Eng., vol. 33, no. 2, pp. 198-209, Apr. 2008.

[8] P. A. van Walree and G. Leus, "Robust underwater telemetry with adaptive turbo multiband equalization," IEEE J. Ocean. Eng., vol. 34, no. 4, pp. 645-655, Oct. 2009.

[9] B. Li et al., "MIMO-OFDM for high-rate underwater acoustic communications," IEEE J. Ocean. Eng., vol. 34, no. 4, pp. 634-644, Oct. 2009

[10] Z. Wang, S. Zhou, G. B. Giannakis, C. R. Berger, and J. Huang, "Frequency-domain oversampling for zeropadded OFDM in underwater acoustic communications," IEEE J. Ocean. Eng., vol. 37, no. 1, pp. 14-24, Jan. 2012.

[11] G. Zhang and H. Dong, "Experimental assessment of a multicarrier underwater acoustic communication system," Appl. Acoust., vol. 72, no. 12, pp. 953-961, Dec. 2011.

[12] G. Zhang and H. Dong, "Spatial diversity in multichannel processing for underwater acoustic communications," Ocean. Eng., vol. 38, nos. 14-15, pp. 1611-1623, Oct. 2011.

[13] S. Roy, T. Duman, L. Ghazikhanian, V. McDonald, J. Proakis, and J. Zeidler, "Enhanced underwater acoustic communication performance using spacetime coding and processing," in Proc. IEEE OCEANS Conf, vol. 1, Nov. 2004.

[14] M. L. Nordenvaad and T. Oberg, "Iterative reception for acoustic underwater MIMO communications," in Proc. IEEE OCEANS Conf.,Sep. 2006,

[15] D. B. Kilfoyle, J. C. Preisig, and A. B. Baggeroer, "Spatial modulation experiments in the underwater acoustic channel," IEEE J. Ocean. Eng.,vol. 30, no. 2, pp. 406-415, Apr. 2005.

[16] E. M. Sozer, M. Stojanovic, and J. G. Proakis, "Underwater acoustic networks," IEEE J. Ocean. Eng., vol. 25, no. 1, pp. 72-83, Jan. 2000.

[17] M. K. Park and V. Rodoplu, "UWAN-MAC: An energy-efficient MAC protocol for underwater acoustic wireless sensor networks," IEEE J. Ocean Eng., vol. 32, no. 3, pp. 710-720, Jul. 2007.

[18] X. Guo, M. R. Frater, and M. J. Ryan, "Design of a propagation delay tolerant MAC protocol for underwater acoustic sensor networks," IEEE J. Ocean. Eng., vol. 34, no. 2, pp. 170-180, Apr. 2009.

[19] W.-H. Liao and C.-C. Huang, "SF-MAC: A spatially fair MAC protocol for underwater acoustic sensor networks," IEEE Sensors J., vol. 12,no. 6, pp. 16861694, Jun. 2012.
[20] M. Zorzi, P. casari and A.F. Harris, "An energyefficient routing schemes for underwater acoustic networks," IEEE J.sel. commmun., vol. 26, no. 9, pp. 1754-1766, Dec. 2008.

[21] J. M. Jordet, M. Stojanovic, and M. Zorzi, "On joint frequency and power allocation in a cross-layer protocol for underwater acoustic networks," IEEE $J$. Ocean. Eng., vol. 35, no. 4, pp. 936-947, Oct. 2010.

[22] R. Niu, M. Moore, D. Klamer, "Decision Fusion in a Wireless Sensor Network with a Large Number of Sensors," Electrical Engineering and Computer Science, paper 82, 2004

[23] B. Chen, L. Tong, and P. K. Varshney, "Channelaware distributed detection in wireless sensor networks," IEEE Signal Process. Mag., vol. 23, no. 4, pp. 16-26, Jul. 2006

[24] B. Chen, R. Jiang, T. Kasetkasem, and P. K Varshney, "Channel aware decision fusion in wireless sensor networks," IEEE Trans. Signal Process, vol. 52, no. 12, pp. 3454-3458, Dec. 2004.

[25] J. Park, E. Kim, and K. Kim, "Large signal robustness of the Chair-Varshney fusion rule under generalizedGaussian noises,"IEEE Sensors J., vol. 10, no. 9, pp. 1438-1439, Sep. 2010.

[26] Lei and R. Schober, "Coherent max-log decision fusion in wireless sensor networks," IEEE Trans. Commun., vol. 58, no. 5, pp. 1327-1332, May 2010.

[27] D. Ciuonzo, G. Romano, and P. Salvo Rossi, "Channel-aware decision fusion in distributed MIMO wireless sensor networks: Decode-and-fuse vs. decode-then-fuse," IEEE Trans. Wireless Commun., vol. 11, no. 8, pp. 2976-2985, Aug. 2012.

[28] D. Ciuonzo, G. Romano, and P. Salvo Rossi, "Performance analysis and design of maximum ratio combining in channel-aware MIMO decision fusion," IEEE Trans. Wireless Commun., vol. 12, no. 9, pp. 4716 4728, Sep. 2013.

[29] D. Ciuonzo, G. Romano, and P. Salvo Rossi, "Optimality of received energy in decision fusion over Rayleigh fading diversity MAC with nonidentical sensors," IEEE Trans. Signal Process, vol. 61, no. 1, pp. 22-27, Jan. 2013.

[30] P. S. Rossi, D.Ciuonzo, T.Ekman, and H. Dong, "Energy Detection for MIMO Decision Fusion in Underwater Sensor Networks" IEEE SENSORS JOURNAL, VOL. 15, NO. 3 MARCH 2015.

[31] S. M. Kay, Fundamental of Statistical Signal processing: Detection Theory, vol. 2. Englewood Cliffs, NJ, USA: Prentice-Hall, 1998.

[32] F. Li, J. S. Evans, and S. Dey, "Decision fusion over non-coherent fading multi-access channels," IEEE Trans. Signal Process, vol. 59, no. 9, pp. 4367-4380, Sep. 2011.

[33] D. Ciuonzo, G. Romano, and P. Salvo Rossi, "Optimality of received energy in decision fusion over Rayleigh fading diversity MAC with nonidentical sensors," IEEE Trans. Signal Process, vol. 61, no. 1, pp. 22-27,Jan. 2013. 